\title{
Bicomplex formulation and Moyal deformation of $(2+1)$-dimensional Fordy-Kulish systems
}

\author{
A. Dimakis ${ }^{1}$ and F. Müller-Hoissen ${ }^{2}$ \\ ${ }^{1}$ Department of Mathematics, University of the Aegean, GR-83200 Karlovasi, Greece, \\ dimakis@aegean.gr \\ 2 Max-Planck-Institut für Strömungsforschung, Bunsenstrasse 10, D-37073 Göttingen, \\ fmuelle@gwdg.de
}

\begin{abstract}
Using bicomplex formalism we construct generalizations of Fordy-Kulish systems of matrix nonlinear Schrödinger equations on two-dimensional space-time in two respects. Firstly, we obtain corresponding equations in three space-time dimensions. Secondly, a Moyal deformation is applied to the space-time coordinates and the ordinary product of functions replaced by the Moyal product in a suitable way. Both generalizations preserve the existence of an infinite set of conservation laws.
\end{abstract}

\section{Introduction}


linear maps d, $\delta: M^{s} \rightarrow M^{s+1}$ satisfying

$$
\mathrm{d}^{2}=0, \quad \delta^{2}=0, \quad \mathrm{~d} \delta+\delta \mathrm{d}=0 .
$$

Associated with a bicomplex is the linear equation

$$
\delta \chi=\lambda \mathrm{d} \chi
$$

where $\chi \in M^{0}$ and $\lambda$ is a parameter [1]. If it admits a (non-trivial) solution as a (formal) power series $\chi=\sum_{r \geq 0} \lambda^{r} \chi^{(r)}$ in $\lambda$, the linear equation leads to

$$
\delta \chi^{(0)}=0, \quad \delta \chi^{(r)}=\mathrm{d} \chi^{(r-1)}, \quad r=1, \ldots, \infty .
$$

As a consequence, $J^{(r+1)}=\mathrm{d} \chi^{(r)}, r=0, \ldots, \infty$, are $\delta$-exact. These elements of $M^{1}$ may be regarded as generalized conserved currents [1].

In section 2 we start with a trivial bicomplex. A certain "dressing" (in the sense of [1]) then leads to a bicomplex formulation of the Fordy-Kulish systems [2] of matrix nonlinear 
Schrödinger equations (matrix-NLS). 1 More precisely, our approach leads to an extension of the latter systems from two to three space-time coordinates $t, x, y$ from which the matrixNLS equations are obtained via the reduction $y=x$. . $^{2}$ Our $(2+1)$-dimensional systems turn out to be matrix generalizations of a system studied in [7, 8] (see also the references given there).

Furthermore, in section 3 deformation quantization [9] is applied to the space-time coordinates. The ordinary commutative product in the algebra $\mathcal{A}$ of smooth functions on $\mathbb{R}^{3}$ is replaced with the $*$-product which is defined by

$$
f * h=\mathbf{m} \circ e^{i \mathcal{P} / 2}(f \otimes h)
$$

where $\mathbf{m}(f \otimes h)=f h$ for all $f, h \in \mathcal{A}$, and $\mathcal{P}: \mathcal{A} \otimes \mathcal{A} \rightarrow \mathcal{A} \otimes \mathcal{A}$ is given by

$$
\mathcal{P}=\vartheta_{1}\left(\partial_{t} \otimes \partial_{x}-\partial_{x} \otimes \partial_{t}\right)+\vartheta_{2}\left(\partial_{t} \otimes \partial_{y}-\partial_{y} \otimes \partial_{t}\right)+\vartheta_{3}\left(\partial_{x} \otimes \partial_{y}-\partial_{y} \otimes \partial_{x}\right)
$$

with real deformation parameters $\vartheta_{j}, j=1,2,3$. Under complex conjugation, we have $\overline{f * h}=\bar{h} * \bar{f}$ for functions $f, h$. The partial derivatives $\partial_{t}, \partial_{x}, \partial_{y}$ are derivations of the $*-$ product. Space-time deformation quantization has been applied recently to various integrable models in [10, 11, 12], for example.

Section 4 deals with the conservation laws of the extended and deformed Fordy-Kulish systems. Section 5 gives corresponding generalized ferromagnet equations for the latter systems. Section 6 contains some conclusions.

\section{Bicomplex formulation of extended Fordy-Kulish sys- tems}

We choose the bicomplex space as $M=M^{0} \otimes \Lambda$ where $M^{0}=C^{\infty}\left(\mathbb{R}^{3}, \mathbb{C}^{N}\right)$ denotes the set of smooth maps $\phi: \mathbb{R}^{3} \rightarrow \mathbb{C}^{N}$ and $\Lambda=\mathbb{C} \oplus \Lambda^{1} \oplus \Lambda^{2}$ is the exterior algebra of a 2-dimensional complex vector space with basis $\tau, \xi$ of $\Lambda^{1}$ (so that $\tau^{2}=\xi^{2}=\tau \xi+\xi \tau=0$ ). $M$ becomes a bicomplex with the maps $\mathrm{d}$ and $\delta$ defined by

$$
\begin{aligned}
\mathrm{d} \phi & =\phi_{t} \tau+\phi_{x} \xi, \\
\delta \phi & =\phi_{y} \tau+(A-a I) \phi \xi
\end{aligned}
$$

where an index denotes a partial derivative with respect to one of the coordinates $t, x, y$ on $\mathbb{R}^{3}$, e.g., $\phi_{t}=\partial_{t} \phi . A$ is a constant $N \times N$ matrix, $I$ the identity matrix, and $a \in \mathbb{C}$. I By

\footnotetext{
${ }^{1}$ See also [3]. For some other generalizations of the NLS equation see [4], in particular.

${ }^{2} \mathrm{~A}$ different extension of the Fordy-Kulish systems to $2+1$ dimensions obtained by replacing the spectral parameter in the $(1+1)$-dimensional systems by a new partial derivative appeared in [5]. See also [6] for some related work.

${ }^{3}$ Only one of these parameters is actually independent since $\mathcal{P}$ is antisymmetric and thus degenerate in three dimensions.

${ }^{4}$ More precisely, $\mathrm{d}^{2}$ vanishes identically, $\delta^{2}=0$ requires $(A-a I)_{y}=0$, and $\mathrm{d} \delta+\delta \mathrm{d}=0$ is satisfied iff $(A-a I)_{t}=0$. This still allows an $x$-dependence of $A-a I$. In the following, we will be interested in the possibility of a reduction of the system to two space-time dimensions by setting $y=x$. Then $A-a I$ has to be constant.
} 
linearity and $\mathrm{d}(\phi \tau+\varphi \xi)=(\mathrm{d} \phi) \tau+(\mathrm{d} \varphi) \xi$ (and correspondingly for $\delta$ ) the maps $\mathrm{d}$ and $\delta$ extend to the whole of $M$. Now we apply a "dressing" to $\mathrm{d}$ as follows,

$$
\begin{aligned}
\mathrm{D} \phi & =\mathrm{d} \phi+\delta(L \phi)-L \delta \phi \\
& =\left(\phi_{t}+L_{y} \phi\right) \tau+\left(\phi_{x}+[A, L] \phi\right) \xi
\end{aligned}
$$

with an $N \times N$ matrix $L$ and $[A, L]=A L-L A$. Besides $\delta^{2}=0$, also $\delta \mathrm{D}+\mathrm{D} \delta=0$ is identically satisfied. The only nontrivial new bicomplex equation is $\mathrm{D}^{2}=0$ which takes the form

$$
L_{y x}-\left[A, L_{t}\right]-\left[L_{y},[A, L]\right]=0
$$

Let us assume that $A$ and $L$ take values in a representation of the Lie algebra $\mathfrak{g}$ of a simple Lie group $G$. Let $K$ be a subgroup of $G$ with Lie algebra $\mathfrak{k}$, and $\mathfrak{m}$ the vector space complement of $\mathfrak{k}$ in $\mathfrak{g}$, so that $\mathfrak{g}=\mathfrak{k} \oplus \mathfrak{m}$ and $[\mathfrak{k}, \mathfrak{k}] \subset \mathfrak{k}$. We assume that the homogeneous space $G / K$ is reductive and moreover symmetric, i.e.,

$$
[\mathfrak{k}, \mathfrak{m}] \subset \mathfrak{m}, \quad[\mathfrak{m}, \mathfrak{m}] \subset \mathfrak{k}
$$

For a Hermitian symmetric space with a complex structure $J: \mathfrak{m} \rightarrow \mathfrak{m}, J^{2}=-1$, the following conditions hold (cf [2]). There is an element $A \in \mathfrak{g}$ such that $\mathfrak{k}=\operatorname{ker}$ ad $A$. For a particular scaling of $A$, we have $J=\operatorname{ad} A$ and there is a subset $\theta^{+}$of the positive root system such that $\mathfrak{m}=\operatorname{span}\left\{e_{ \pm \alpha} \mid \alpha \in \theta^{+}\right\}$and $\left[A, e_{ \pm \alpha}\right]= \pm i e_{ \pm \alpha}$ for $\alpha \in \theta^{+}$. Here $e_{\alpha}$ belongs to the Cartan-Weyl basis. Moreover, $\left[e_{\alpha}, e_{\beta}\right]=0=\left[e_{-\alpha}, e_{-\beta}\right]$ for all $\alpha, \beta \in \theta^{+}$.

Now we choose $A$ in (2.2) with the above properties. $A$ is then $\mathfrak{k}$-valued. With the decomposition

$$
L=Q+P
$$

where $Q \in \mathfrak{m}$ and $P \in \mathfrak{k}$, the $\mathfrak{k}$ - and $\mathfrak{m}$-part of (2.4) reads, respectively,

$$
\begin{aligned}
P_{x y} & =\left[Q_{y},[A, Q]\right] \\
Q_{x y} & =[A, Q]_{t}+\left[P_{y},[A, Q]\right] .
\end{aligned}
$$

According to the above assumptions, $Q$ has a decomposition

$$
Q=Q^{+}+Q^{-}, \quad\left[A, Q^{ \pm}\right]= \pm i Q^{ \pm}
$$

Now one finds that (2.7) can be integrated with respect to $y$. This yields

$$
P_{x}=-i\left[Q^{+}, Q^{-}\right]
$$

(up to addition of a $\mathfrak{k}$-valued matrix which only depends on $t$ and $x$ and which we disregard in the following). (2.8) now leads to

$$
i Q_{t}^{ \pm} \mp Q_{x y}^{ \pm}+i\left[P_{y}, Q^{ \pm}\right]=0 .
$$


The system of equations (2.10), (2.11) constitutes an extension of the Fordy-Kulish systems [2] to which it reduces when $y=x$. In the latter case, (2.10) determines $P_{x}$ which can then be eliminated from (2.11). We are then left with the following equations,

$$
i Q_{t}^{ \pm} \mp Q_{x x}^{ \pm}+\left[\left[Q^{+}, Q^{-}\right], Q^{ \pm}\right]=0 .
$$

Example. Let $G=S U(2)$ and $y=x$. The subalgebra of $s u(2)$ spanned by $A=(i / 2) \sigma_{3}$ with the Pauli matrix $\sigma_{3}$ is clearly annihilated by ad $A$ and generates a $U(1)$ subgroup. Let

$$
Q^{+}=\left(\begin{array}{cc}
0 & \psi \\
0 & 0
\end{array}\right), \quad Q^{-}=\left(\begin{array}{cc}
0 & 0 \\
-\bar{\psi} & 0
\end{array}\right)
$$

where $\psi$ is a complex function with complex conjugate $\bar{\psi}$. Then the two equations (2.12) both reduce to the nonlinear Schrödinger equation

$$
i \psi_{t}=-\psi_{x x}-2|\psi|^{2} \psi
$$

This example is easily generalized [2]. Let us consider the Hermitian symmetric space $S U(N) / S(U(n) \times U(N-n))$ and choose

$$
A=\left(\begin{array}{cc}
c_{1} I_{n} & 0 \\
0 & c_{2} I_{N-n}
\end{array}\right)
$$

where $I_{n}$ is the $n \times n$ unit matrix and $c_{1}, c_{2} \in \mathbb{C}$. With

$$
Q^{+}=\left(\begin{array}{cc}
0 & q \\
0 & 0
\end{array}\right), \quad Q^{-}=\left(\begin{array}{cc}
0 & 0 \\
-q^{\dagger} & 0
\end{array}\right)
$$

where $q$ is an $n \times(N-n)$ matrix with Hermitian conjugate $q^{\dagger}$, we get

$$
\left[A, Q^{ \pm}\right]= \pm\left(c_{1}-c_{2}\right) Q^{ \pm}
$$

The constants $c_{1}, c_{2}$ are thus related by $c_{1}-c_{2}=i$. Since $A$ must be traceless, we also have $n c_{1}+(N-n) c_{2}=0$. Hence

$$
c_{1}=\frac{N-n}{N} i, \quad c_{2}=-\frac{n}{N} i
$$

The matrix $P$ must have the form

$$
P=\left(\begin{array}{ll}
p & 0 \\
0 & r
\end{array}\right)
$$

with an $n \times n$ matrix $p$ and an $(N-n) \times(N-n)$ matrix $r$. From (2.10) we obtain the equations

$$
p_{x}=i q q^{\dagger}, \quad r_{x}=-i q^{\dagger} q
$$


which are compatible with the unitarity constraints $p^{\dagger}=-p$ and $r^{\dagger}=-r$, and with $\operatorname{tr}(p)+$ $\operatorname{tr}(r)=0$. (2.11) becomes

$$
i q_{t}-q_{x y}+i\left(p_{y} q-q r_{y}\right)=0
$$

and its Hermitian conjugate. The reduction $y=x$ leads to the matrix nonlinear Schrödinger equation [2]

$$
i q_{t}-q_{x x}-2 q q^{\dagger} q=0 .
$$

The more general systems determined by (2.20) and (2.21) on three-dimensional space-time will be called "extended matrix-NLS equations" in the following.

Other examples of Hermitian symmetric spaces lead to further matrix nonlinear Schrödinger equations [2] and extensions in the above sense.

\section{Space-time deformation quantization of the extended matrix-NLS equations}

In this section we apply a deformation quantization to the algebra of (smooth) functions on space-time. The bicomplex $(M, \mathrm{D}, \delta)$ introduced in the previous section then generalizes to the deformed noncommutative algebra with the following definition,

$$
\begin{aligned}
\mathrm{D} \phi & =\mathrm{d} \phi+\delta(L * \phi)-L * \delta \phi \\
& =\left(\phi_{t}+L_{y} * \phi\right) \tau+\left(\phi_{x}+[A, L]_{*} * \phi\right) \xi
\end{aligned}
$$

with $[A, L]_{*}=A * L-L * A$. The only nontrivial bicomplex equation is still $\mathrm{D}^{2}=0$ which now takes the form

$$
L_{y x}-\left[A, L_{t}\right]_{*}-\left[L_{y},[A, L]_{*}\right]_{*}=0 .
$$

Of course, the $*$-commutator does not preserve a Lie algebra structure, in general. As a consequence, a decomposition of the last equation like that worked out for Hermitian symmetric spaces in [2] and the previous section does not work, in general. However, in the case of extended matrix-NLS equations only a certain block structure of the matrices entering the bicomplex maps is important. Let $\mathfrak{m}^{ \pm}$be the set of all $N \times N$ matrices of the form of $Q^{ \pm}$in (2.16). Let $\mathfrak{k}$ be the set of all block diagonal matrices (like $P$ in (2.19)). Then we have $\mathfrak{k} * \mathfrak{k} \subset \mathfrak{k}, \mathfrak{k} * \mathfrak{m}^{ \pm} \subset \mathfrak{m}^{ \pm}$and $\mathfrak{m}^{ \pm} * \mathfrak{k} \subset \mathfrak{m}^{ \pm}$. Moreover, since $A$ given in (2.15) with (2.18) is constant, we still have $[A, P]_{*}=0$ and $\left[A, Q^{ \pm}\right]_{*}= \pm i Q^{ \pm}$. Hence, we can proceed with the decomposition $L=Q^{+}+Q^{-}+P$ as in the previous section. The above deformed bicomplex equation now results in the following system,

$$
p_{x}=i q * q^{\dagger}, \quad r_{x}=-i q^{\dagger} * q
$$

and

$$
i q_{t}-q_{x y}+i\left(p_{y} * q-q * r_{y}\right)=0 .
$$


This is a noncommutative version of the corresponding extended matrix-NLS system. The equations (3.3) are consistent with unitarity constraints on $p$ and $r$, but not with $\operatorname{tr}(p)+$ $\operatorname{tr}(r)=0$. In contrast to the classical case, these matrices can no longer be taken as Lie algebra valued. They have values in the corresponding enveloping algebra instead.

The reduction $y=x$ of the above system leads to the noncommutative matrix nonlinear Schrödinger equation

$$
i q_{t}-q_{x x}-2 q * q^{\dagger} * q=0
$$

which is the matrix version of the noncommutative nonlinear Schrödinger equation treated in 11 .

\section{Conservation laws for the three-dimensional exten- sions and deformations of matrix-NLS equations}

The linear equation associated with the bicomplex underlying the deformed extended matrixNLS equations of the previous section is

$$
\delta \chi=\lambda \mathrm{D} \chi
$$

with a parameter $\lambda . \chi$ is taken to be an $N \times n$ matrix of functions. The linear equation is equivalent to the two equations

$$
\begin{aligned}
\chi_{y} & =\lambda\left(\chi_{t}+L_{y} * \chi\right) \\
(A-a) \chi & =\lambda\left(\chi_{x}+[A, L]_{*} * \chi\right) .
\end{aligned}
$$

Let us decompose $\chi$ into an $n \times n$ matrix $\alpha$ and an $(N-n) \times n$ matrix $\beta$,

$$
\chi=\left(\begin{array}{c}
\alpha \\
\beta
\end{array}\right) .
$$

In order to have a nontrivial solution of $\delta \chi^{(0)}=0$, we choose $a$ as an eigenvalue of $A$. To be more concrete,

$$
a=\frac{N-n}{N} i, \quad A-a I=-i\left(\begin{array}{cc}
0 & 0 \\
0 & I_{N-n}
\end{array}\right), \quad \chi^{(0)}=\left(\begin{array}{c}
I_{n} \\
0
\end{array}\right) .
$$

From (4.3) we get

$$
\alpha_{x}+i q * \beta=0, \quad \beta=i \lambda\left(\beta_{x}+i q^{\dagger} * \alpha\right) .
$$

Assuming that $q$ has a left $*$-inverse, this implies

$$
\begin{aligned}
\beta & =i q_{*}^{-1} * \alpha_{x}, \\
\alpha_{x} & =i \lambda\left(\alpha_{x x}-q_{x} * q_{*}^{-1} * \alpha_{x}+q * q^{\dagger} * \alpha\right) .
\end{aligned}
$$


Furthermore, (4.2) leads to

$$
\alpha_{y}=\lambda\left(\alpha_{t}+p_{y} * \alpha+i q_{y} * q^{-1} * \alpha_{x}\right) .
$$

$\alpha$ has a right $*$-inverse at least as a formal power series in $\lambda$, since at 0 th order it equals $I_{n}$. Hence there are $n \times n$ matrices $\rho, \sigma$ and $\zeta$ such that

$$
\alpha_{x}=i \lambda \rho * \alpha, \quad \alpha_{t}=i \lambda \sigma * \alpha, \quad \alpha_{y}=i \lambda \zeta * \alpha .
$$

Then (4.8) and (4.9) lead, respectively, to

$$
\begin{aligned}
\rho & =q * q^{\dagger}+i \lambda\left(\rho_{x}-q_{x} * q_{*}^{-1} * \rho\right)-\lambda^{2} \rho * \rho, \\
\lambda \sigma-\zeta & =i p_{y}-i \lambda q_{y} * q_{*}^{-1} * \rho .
\end{aligned}
$$

The integrability conditions $\alpha_{x t}=\alpha_{t x}$ and $\alpha_{x y}=\alpha_{y x}$ together with (4.10) yield

$$
\begin{aligned}
\rho_{t}-\sigma_{x}+i \lambda[\rho, \sigma]_{*} & =0, \\
\zeta_{x}-\rho_{y}-i \lambda[\rho, \zeta]_{*} & =0 .
\end{aligned}
$$

Differentiation of (4.12) with respect to $x$, using (4.14), $i p_{x}=-q * q^{\dagger}(\mathrm{cf}(3.3))$ and (4.11), leads to

$$
\sigma_{x}=\left(i\left(\rho_{x}-q_{x} * q_{*}^{-1} * \rho\right)-\lambda \rho * \rho\right)_{y}-i\left(q_{y} * q_{*}^{-1} * \rho\right)_{x}+i[\rho, \zeta]_{*} .
$$

Inserted in (4.13), this yields

$$
\rho_{t}+i\left(q_{y} * q_{*}^{-1} * \rho\right)_{x}-\left(i\left(\rho_{x}-q_{x} * q_{*}^{-1} * \rho\right)-\lambda \rho * \rho\right)_{y}+i[\rho, \lambda \sigma-\zeta]_{*}=0 .
$$

In terms of the product

$$
f \diamond h=\mathbf{m} \circ \frac{\sin (\mathcal{P} / 2)}{\mathcal{P} / 2}(f \otimes h)
$$

with $\mathcal{P}$ defined in (1.5), the *-commutator of two functions can be written as follows,

$$
\begin{aligned}
\frac{1}{i}[f, h]_{*} & =2 \mathbf{m} \circ \sin (\mathcal{P} / 2)(f \otimes h) \\
& =\left(f \diamond\left(\vartheta_{1} h_{x}+\vartheta_{2} h_{y}\right)\right)_{t}+\left(f \diamond\left(-\vartheta_{1} h_{t}+\vartheta_{3} h_{y}\right)\right)_{x}-\left(f \diamond\left(\vartheta_{2} h_{t}+\vartheta_{3} h_{x}\right)\right)_{y}
\end{aligned}
$$

Taking the trace of (4.16), using the last formula and (4.12), we obtain the conservation law

$$
\begin{aligned}
0= & \operatorname{tr}\left(\rho-i \rho \diamond\left[\left(\vartheta_{1} \partial_{x}+\vartheta_{2} \partial_{y}\right)\left(p_{y}-\lambda q_{y} * q_{*}^{-1} * \rho\right)\right]\right)_{t} \\
& +\operatorname{tr}\left(i q_{y} * q_{*}^{-1} * \rho+i \rho \diamond\left[\left(\vartheta_{1} \partial_{t}-\vartheta_{3} \partial_{y}\right)\left(p_{y}-\lambda q_{y} * q_{*}^{-1} * \rho\right)\right]\right)_{x} \\
& +\operatorname{tr}\left(\lambda \rho * \rho-i\left(\rho_{x}-q_{x} * q_{*}^{-1} * \rho\right)+i \rho \diamond\left[\left(\vartheta_{2} \partial_{t}+\vartheta_{3} \partial_{x}\right)\left(p_{y}-\lambda q_{y} * q_{*}^{-1} * \rho\right)\right]\right)_{y}
\end{aligned}
$$

Expanding $\rho$ in a formal power series in $\lambda$, i.e.,

$$
\rho=\sum_{r=0}^{\infty} \lambda^{r} \rho^{(r)}
$$


(4.11) leads to

$$
\rho^{(0)}=q * q^{\dagger}, \quad \rho^{(1)}=i q * q_{x}^{\dagger}, \quad \rho^{(2)}=-q * q^{\dagger} * q * q^{\dagger}-q * q_{x x}^{\dagger}
$$

and

$$
\rho^{(r)}=i\left(\rho_{x}^{(r-1)}-q_{x} * q_{*}^{-1} * \rho^{(r-1)}\right)-\sum_{s=0}^{r-2}\left(\begin{array}{c}
r-2 \\
s
\end{array}\right) \rho^{(s)} * \rho^{(r-2-s)}
$$

for $r \geq 2$. Inserting this in the expression

$$
w=\sum_{r=0}^{\infty} \lambda^{r} w^{(r)}=\operatorname{tr}\left(\rho-i \rho \diamond\left[\left(\vartheta_{1} \partial_{x}+\vartheta_{2} \partial_{y}\right)\left(p_{y}-\lambda q_{y} * q_{*}^{-1} * \rho\right)\right]\right)
$$

which appears in the above conservation law, an infinite set of conserved densities is obtained, starting with

$$
\begin{aligned}
w^{(0)}= & \operatorname{tr}\left(q * q^{\dagger}-i\left(q * q^{\dagger}\right) \diamond\left[\left(\vartheta_{1} \partial_{x}+\vartheta_{2} \partial_{y}\right) p_{y}\right]\right) \\
w^{(1)}= & \operatorname{tr}\left(i q * q_{x}^{\dagger}+\left(q * q_{x}^{\dagger}\right) \diamond\left[\left(\vartheta_{1} \partial_{x}+\vartheta_{2} \partial_{y}\right) p_{y}\right]\right. \\
& \left.+i\left(q * q^{\dagger}\right) \diamond\left[\left(\vartheta_{1} \partial_{x}+\vartheta_{2} \partial_{y}\right)\left(q_{y} * q^{\dagger}\right)\right]\right) \\
w^{(2)}= & \operatorname{tr}\left(-q * q^{\dagger} * q * q^{\dagger}-q * q_{x x}^{\dagger}+i\left(q * q^{\dagger} * q * q^{\dagger}+q * q_{x x}^{\dagger}\right) \diamond\left[\left(\vartheta_{1} \partial_{x}+\vartheta_{2} \partial_{y}\right) p_{y}\right]\right. \\
& \left.-\left(q * q_{x}^{\dagger}\right) \diamond\left[\left(\vartheta_{1} \partial_{x}+\vartheta_{2} \partial_{y}\right)\left(q_{y} * q^{\dagger}\right)\right]-\left(q * q^{\dagger}\right) \diamond\left[\left(\vartheta_{1} \partial_{x}+\vartheta_{2} \partial_{y}\right)\left(q_{y} * q_{x}^{\dagger}\right)\right]\right)
\end{aligned}
$$

which in turn can be expanded in (formal) power series in the deformation parameters. For vanishing deformation parameters, the conserved densities $w^{(r)}$ are polynomials in $q, q^{\dagger}$ and their $x$-derivatives, but no $y$-derivatives. This means that the conserved densities of an extended matrix-NLS system are the same (as polynomials in the fields and their partial derivatives) as those of the corresponding matrix-NLS system (which is obtained from the former by setting $y=x$ ). This is no longer so after deformation.

\section{Generalized ferromagnet equations associated with deformed extended Fordy-Kulish systems}

A gauge transformation of the (deformed) bicomplex considered in section 3 is a map $g$ : $\mathbb{R}^{3} \rightarrow G$ such that

$$
\mathrm{D} \phi \mapsto \mathrm{D}^{\prime} \phi^{\prime}=g_{*}^{-1} \mathrm{D}(g * \phi), \quad \delta \phi \mapsto \delta^{\prime} \phi^{\prime}=g_{*}^{-1} \delta(g * \phi)
$$

for all $\phi \in M$ with $\phi^{\prime}=g * \phi$. Such a map leaves the bicomplex equations invariant. Let us choose $g$ such that $\mathrm{D}^{\prime}=\mathrm{d}$. Then

$$
g_{*}^{-1} * g_{t}=-g_{*}^{-1} * L_{y} * g, \quad g_{*}^{-1} * g_{x}=-g_{*}^{-1} *[A, L]_{*} * g
$$

\footnotetext{
${ }^{5}$ These expressions do not involve the $*$-inverse of $q$ and also apply to solutions for which $q$ is not $*$ invertible.
} 
and, writing $\mathcal{D}$ instead of $\delta^{\prime}$, we get

$$
\mathcal{D} \phi=\left(\phi_{y}+R * \phi\right) \tau+(S-a I) * \phi \xi
$$

where we introduced the abbreviations

$$
R=g_{*}^{-1} * g_{y}, \quad S=g_{*}^{-1} * A * g .
$$

Now $\mathcal{D}^{2}=0$ reads

$$
S_{y}=[S, R]_{*}
$$

and $\mathrm{d} \mathcal{D}+\mathcal{D} \mathrm{d}=0$ becomes

$$
S_{t}=R_{x}
$$

Decomposing $R$ as follows,

$$
R=U+W,
$$

where $g * U * g_{*}^{-1} \in \mathfrak{m}$ and $g * W * g_{*}^{-1} \in \mathfrak{k}$, we find $[S, R]_{*}=[S, U]_{*}$ and thus $\left[S, S_{y}\right]_{*}=$ $\left[S,[S, U]_{*}\right]_{*}$. Using $(\operatorname{ad} A)^{2}=-I$ which implies $(\operatorname{ad} S)^{2}=-I$, we get $\left[S, S_{y}\right]_{*}=-U$ so that (5.6) can be written as follows,

$$
S_{t}=-\left(\left[S, S_{y}\right]_{*}-W\right)_{x} .
$$

Furthermore, (5.7) becomes

$$
g_{*}^{-1} * g_{y}=-\left[S, S_{y}\right]_{*}+W .
$$

(5.4) leads to $S_{x}=\left[S, g_{*}^{-1} * g_{x}\right]_{*}$ (since $A$ is constant) which implies

$$
g_{*}^{-1} * g_{x}=-\left[S, S_{x}\right]_{*}
$$

by use of $(\operatorname{ad} S)^{2}=-I$. Together with the identity

$$
\left(g_{*}^{-1} * g_{x}\right)_{y}-\left(g_{*}^{-1} * g_{y}\right)_{x}=\left[g_{*}^{-1} * g_{x}, g_{*}^{-1} * g_{y}\right]_{*}
$$

the last two equations lead to

$$
W_{x}=\left[\left[S, S_{x}\right]_{*}, W\right]_{*}+2\left[S_{x}, S_{y}\right]_{*}-\left[\left[S, S_{x}\right]_{*},\left[S, S_{y}\right]_{*}\right]_{*} .
$$

The second term on the rhs can be rewritten as follows,

$$
\begin{aligned}
{\left[\left[S, S_{x}\right]_{*},\left[S, S_{y}\right]_{*}\right]_{*} } & =-\left[S,\left[\left[S, S_{x}\right]_{*}\right]_{*}, S_{y}\right]_{*}+\left[S,\left[\left[S, S_{x}\right]_{*}, S_{y}\right]_{*}\right]_{*} \\
& =\left[S_{x}, S_{y}\right]_{*}+\left[S,\left[\left[S, S_{x}\right]_{*}, S_{y}\right]_{*}\right]_{*}
\end{aligned}
$$


using again $(\operatorname{ad} S)^{2}=-I$. (5.2) implies $g_{*}^{-1} * g_{x} \in g_{*}^{-1} * \mathfrak{m} * g$ and thus $\left[S, S_{x}\right]_{*} \in g_{*}^{-1} * \mathfrak{m} * g$. It follows that $\left[\left[S, S_{x}\right]_{*}, S_{y}\right]_{*} \in g_{*}^{-1} * \mathfrak{k} * g$. Since $S$ commutes with all elements of $g_{*}^{-1} * \mathfrak{k} * g$, we have $\left[S,\left[\left[S, S_{x}\right]_{*}, S_{y}\right]_{*}\right]_{*}=0$. Hence

$$
W_{x}=\left[S_{x}, S_{y}\right]_{*}+\left[\left[S, S_{x}\right]_{*}, W\right]_{*} .
$$

Together with (5.8) this constitutes a $(2+1)$-dimensional matrix generalization of the Heisenberg ferromagnet equation, as we shall explain below.

Example. For $N=2$ and $n=1$ we have $S^{2}=-\frac{1}{4} I$ and $W=u * S$ with a function $u$. Using

$$
\left[\left[S, S_{x}\right]_{*}, u * S\right]_{*}=\left[\left[S, S_{x}\right]_{*}, u\right]_{*} * S+u *\left[\left[S, S_{x}\right]_{*}, S\right]_{*}=\left[\left[S, S_{x}\right]_{*}, u\right]_{*} * S+u * S_{x},
$$

the system (5.8),(5.14) becomes

$$
S_{t}=-\left(\left[S, S_{y}\right]_{*}-u * S\right)_{x}, \quad u_{x}=-4\left[S_{x}, S_{y}\right]_{*} * S+\left[\left[S, S_{x}\right]_{*}, u\right]_{*} .
$$

In the undeformed (commutative) case, the term $\left[\left[S, S_{x}\right]_{*}, u\right]_{*}$ disappears. Then we recover a system of equations which has been discussed in [7] (see also the references given there). Its nonlinear Schrödinger-type counterpart has been considered in [8]. Our equations (5.8), (5.14) thus constitute a matrix generalization of this system. Let us choose

$$
g=\exp \left(-\frac{i}{2} \sigma_{2} \varphi\right) \exp \left(\frac{i}{2} \sigma_{3} t\right)=\left(\begin{array}{cc}
e^{i t / 2} \cos (\varphi / 2) & -e^{-i t / 2} \sin (\varphi / 2) \\
e^{i t / 2} \sin (\varphi / 2) & e^{-i t / 2} \cos (\varphi / 2)
\end{array}\right)
$$

with a function $\varphi(x, y)$ and the Pauli matrices $\sigma_{2}$ and $\sigma_{3}$, we get

$$
S=\frac{i}{2}\left(\begin{array}{cc}
\cos \varphi & -e^{-i t} \sin \varphi \\
-e^{i t} \sin \varphi & -\cos \varphi
\end{array}\right)
$$

Then $\left[S_{x}, S_{y}\right]=0$ and we can choose $W=0$. Now (5.8) reduces to the sine-Gordon equation

$$
\varphi_{x y}=\sin \varphi \text {. }
$$

For $y=x,(5.2)$ and the decomposition (5.7) imply $W=0$. Without deformation, (5.8) then reduces to $S_{t}=-\left[S, S_{x x}\right]$ which is a matrix generalization [2] (see also [13]) of an equation which describes a one-dimensional continuous spin system (Heisenberg ferromagnet) [14]. Its equivalence with the nonlinear Schrödinger equation was demonstrated in [15] (see also [16]).

With $S=-\gamma_{x},(5.8)$ becomes

$$
\gamma_{t}=\left[\gamma_{x}, \gamma_{x y}\right]_{*}-W
$$

For $y=x$ we have $W=0$. Without deformation, the last equation is then a generalization [2] of the Da Rios equation which describes evolution of a thin vortex filament in a threedimensional fluid [17] (see also [18, 19], in particular). Its equivalence with the nonlinear Schrödinger equation was first shown in [20].

\footnotetext{
${ }^{6}$ An arbitrary matrix $V$ which does not depend on $x$ arises as a "constant of integration". It can be eliminated by a redefinition of $\gamma$.
} 


\section{Conclusions}

Using bicomplex formalism [1], we obtained an extension of the Fordy-Kulish systems of matrix-NLS equations to three space-time dimensions. Moreover, corresponding equations on a noncommutative space-time are obtained by deformation quantization. We have shown that the resulting equations still possess an infinite set of conserved densities. Moreover, there is a gauge-equivalent generalized ferromagnet equation for all of these systems.

The Fordy-Kulish systems generalize the nonlinear Schrödinger equation, respectively the Heisenberg magnet or the Da Rios equation. The latter equations are associated with the simplest Hermitian symmetric space $S U(2) / S(U(1) \times U(1))$ in the series $S U(N) / S(U(n) \times$ $U(N-n))$. The corresponding extended Fordy-Kulish system associated with this space also reproduces the sine-Gordon equation, as shown in the previous section. There may be a way to understand the extended Fordy-Kulish systems as generalizations of the sine-Gordon equation in a similar way as they are quite obvious generalizations of the NLS equation.

Certain limits of string, D-brane and M theory generate field theories on noncommutative space-times which are obtained by a space-time deformation quantization (see 21] and the references given there). Among the various noncommutative models which arise in these and other ways, "integrable" models will certainly be of special interest because of their highly distinguished properties. Although a suitable notion of integrability of a noncommutative field theory is not yet at hand, we think that the existence of an infinite set of conserved densities should be taken as a partial requirement. In case of the deformations considered in this work (see also [11, 12]), this requirement is satisfied.

Acknowledgment. The authors are grateful to Partha Guha for discussions and for drawing their attention to Refs. 2 and 13.

\section{References}

[1] Dimakis A and Müller-Hoissen F 2000 Bi-differential calculi and integrable models, J. Phys. A: Math. Gen. 33 957-974

Dimakis A and Müller-Hoissen F 2000 Bicomplexes and integrable models J. Phys. A: Math. Gen. 33 6579-6591

[2] Fordy A P and Kulish P P 1983 Nonlinear Schrödinger equations and simple Lie algebras Commun. Math. Phys. 89 427-443

[3] Oh P and Park Q-H 1996 More on generalized Heisenberg ferromagnet models Phys. Lett. B $383333-338$

Terng C L and Uhlenbeck K 1999 Schrödinger flows on Grassmannians, math.DG/9901086

[4] Fordy A P 1984 Derivative nonlinear Schrödinger equations and Hermitian symmetric spaces J. Phys. A: Math. Gen. 17 1235-1245

Olver P J and Sokolov V V 1998 Non-abelian integrable systems of the nonlinear 
Schrödinger type Inverse Problems 14 L5-L8

Tsuchida T and Wadati M 1999 Complete integrability of derivative nonlinear Schrödinger equations Inverse Problems 15 1363-1373

Porsezian K 1997 Completely integrable nonlinear Schrödinger type equations on moving space curves Phys. Rev. E 55 3785-3788

Porsezian K 1998 Nonlinear Schrödinger family on moving space curves: Lax pairs, soliton solution and equivalent spin chains Chaos, Solitons \& Fractals 9 1709-1722

[5] Athorne C and Fordy A 1987 Integrable equations in $(2+1)$ dimensions associated with symmetric and homogeneous spaces J. Math. Phys. 28 2018-2024

[6] Ishimori Y 1984 Multi-vortex solutions of a two-dimensional nonlinear wave equation Progr. Theor. Phys. 72 33-37

Cheng Y, Li Y-S and Tang G-X 1990 The gauge equivalence of the Davey-Stewartson equation and $(2+1)$-dimensional continuous Heisenberg ferromagnetic model $J$. Phys. A: Math. Gen. 23 L473-L477

Konopelchenko B G 1993 Solitons in Multidimensions: Inverse Spectral Transform Method (Singapore: World Scientific)

Chakravarty S, Kent S L and Newman E T 1995 Some reductions of the self-dual YangMills equations to integrable systems in $2+1$ dimensions $J$. Math. Phys. 36 763-772 Radha R and Lakshmanan M 1999 Generalized dromions in the $(2+1)$ dimensional long dispersive wave (2LDW) and scalar nonlinear Schrödinger (NLS) equations Chaos, Solitons \& Fractals 10 1821-1824

[7] Myrzakulov R, Vijayalakshmi S, Syzdykova R N and Lakshmanan M 1998 On the simplest $(2+1)$ dimensional integrable spin systems and their equivalent Schrödinger equations J. Math. Phys. 39 2122-2140

Myrzakulov R, Nugmanova G N and Syzdykova R N 1998 Gauge equivalence between $(2+1)$-dimensional continuous Heisenberg ferromagnetic models and nonlinear Schrödinger-type equations J. Phys. A: Math. Gen. 31 9535-9545

Ding Q 1999 The gauge equivalence of the NLS and the Schrödinger flow of maps in $2+1$ dimensions J. Phys. A: Math. Gen. 32 5087-5096

[8] Zakharov V E 1980 The inverse scattering method, in Solitons, eds R K Bullough and P J Caudrey (Berlin: Springer), pp 243-285

Strachan I A B 1993 Some integrable hierarchies in $(2+1)$ dimensions and their twistor description J. Math. Phys. 34 243-259

Myrzakulov R, Vijayalakshmi S, Nugmanova G N and Lakshmanan M 1997 A (2+ 1) dimensional integrable spin model: geometrical and gauge equivalent counterpart, solitons and localized coherent structures Phys. Lett. A 233 391-396

[9] Bayen F, Flato M, Fronsdal C, Lichnerowicz A and Sternheimer D 1978 Deformation theory and quantization I, II, Ann. Phys. 111 61-151

[10] Takasaki K 2000 Anti-self dual Yang-Mills equations on noncommutative spacetime, hep-th/0005194 
[11] Dimakis A and Müller-Hoissen F 2000 A noncommutative version of the nonlinear Schrödinger equation, hep-th/0007015

[12] Dimakis A and Müller-Hoissen F 2000 Bicomplexes, integrable models, and noncommutative geometry, hep-th/0006005

Dimakis A and Müller-Hoissen F 2000 Noncommutative Korteweg-de-Vries equation, hep-th/0007074

Dimakis A and Müller-Hoissen F 2000 Moyal deformation, Seiberg-Witten map, and integrable models, hep-th/0007160, to appear in Lett. Math. Phys.

[13] Langer J and Perline R 2000 Geometric Realizations of Fordy-Kulish systems, to appear in Pacific J. Math.

[14] Lakshmanan M, Ruijgrok Th W and Thompson C J 1976 On the dynamics of a continuum spin system Physica 84A 577-590

[15] Zakharov V E and Takhtajan L A 1979 Equivalence of the nonlinear Schrödinger equation and the equation of a Heisenberg ferromagnet Theor. Math. Phys. 38 17-23

[16] Faddeev L D and Takhtajan L A 1987 Hamiltonian Methods in the Theory of Solitons (Berlin: Springer)

[17] Da Rios L S 1906 Sul moto d'un liquido indefinito con un filetto vorticoso di forma qualunque, Rend. Circ. Mat. Palermo 22 117-135

[18] Ricca R L 1991 Rediscovery of Da Rios equations Nature 352 561-562

[19] Langer J 1999 Recursion in curve geometry New York Journal of Mathematics 5 25-51

[20] Hasimoto H 1972 A soliton on a vortex filament J. Fluid Mech. 51 477-485

[21] Seiberg N and Witten E 1999 String theory and noncommutative geometry JHEP 932 